\title{
NEW METHOD FOR STUDYING BIAXIAL DEFORMATION EFFECTS ON OPTICAL SPECTRA OF QUANTUM WELLS
}

\author{
T.P. Sosin* aNd W. TRZECIAKowsKI \\ High Pressure Research Center "Unipress", Polish Academy of Sciences \\ Sokolowska 29, 01-142 Warszawa, Poland
}

The effects of large deformation were studied by preparing thin $(20-30 \mu \mathrm{m})$ membranes with quantum-well layers on top. A small gas pressure of a few bar deforms the membrane substantially and changes the optical spectra of the quantum wells. We present the results of the photoluminescence and absorption from GaAs/AlGaAs and from InGaAs/GaAs quantum wells subjected to tensile and to compressive biaxial strain. The light-hole lines shift more than two times faster than the heavy-hole lines so that they cross under tensile strain.

PACS numbers: 78.66.Fd, 73.20.Dx

\section{Introduction}

Large deformation of a solid can usually be obtained using large pressures of a few kilobars. This holds both for hydrostatic and for uniaxial experiments. Moreover, such experiments require sophisticated apparatus. Here we propose a simple method that allows to obtain large deformations with a gas pressure of a few bars and does not require any specialized equipment. The method consists in etching a 1-2 mm hole in the sample so that we obtain a membrane about $20 \mu \mathrm{m}$ thick while its edges are $300-500 \mu \mathrm{m}$ thick. When the gas pressure is applied to such membrane we can get large deformations on both surfaces, depending on the point we study (Fig. 1). For example, in the middle of a circular or square membrane the surface layer gets biaxially extended (compressed) on the outside (inside) of the membrane. Close to the edge of the membrane the deformation is uniaxial. If we collect the optical spectra from a small spot on the sample (typically $50 \mu \mathrm{m}$ ) we can scan the surface of a 1-2 mm membrane looking at different stress conditions. The gas pressure can be applied to the membrane using a capillary so that the little cell with the membrane can be easily cooled in a cryostat, put

*Also at the Institute of Physics, Warsaw University of Technology, Koszykowa 75, 00-662 Warszawa, Poland. 


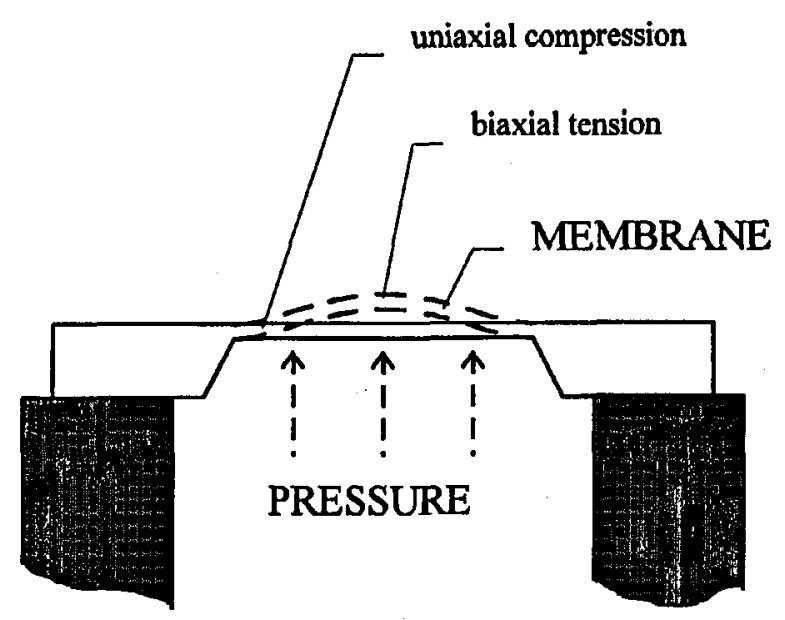

Fig. 1. Schematic cross-section of the semiconductor membrane (not to scale). Dashed lines show the membrane deformed by pressure. Quantum-well layers are on the top surface. The sample can also be mounted upside down so that under pressure we get biaxial compression of the quantum wells in the middle of the membrane.

in the magnetic field etc. and the pressure will be varied in situ. The cell has a sapphire window opposite to the membrane so that we can access both surfaces of the membrane. In the following we will show some results obtained from the middle of the deformed GaAs membrane with quantum well layers close to the surface. Let us first briefly analyze what kind of effects we can expect for biaxially strained quantum wells.

\section{Theory}

In the center of a circular (or square) membrane the deformation tensor is diagonal with $\varepsilon_{x x}=\varepsilon_{y y}=\varepsilon_{0}$ and $\varepsilon_{z z}=-2 \nu \varepsilon_{0} /(1-\nu)$. Here $\varepsilon_{0}$ is the biaxial deformation, $\nu$ is the Poisson ratio. Inserting these values into the Bir and Pikus formulas [1] for the shift of the valence bands at $k=0$ we get

$$
\Delta E\left(\varepsilon_{0}\right)=2 a \varepsilon_{0} \frac{1-2 \nu}{1-\nu} \pm b \varepsilon_{0} \frac{1+\nu}{1-\nu}
$$

where $a$ and $b$ are the hydrostatic and uniaxial deformation potentials, respectively, and the plus (minus) sign corresponds to light (heavy) holes. If we neglect the differences in these potentials and in compressibilities of GaAs and AlGaAs (or InGaAs) we should have the same shifts in the wells and in the barriers. If we also neglect the possible changes of the exciton binding due to deformation, Eq. (1) should represent the expected shifts of the heavy and light-hole lines from the deformed quantum wells. In order to relate $\varepsilon_{0}$ to the external pressure we have to calculate the deformation in the membrane. For circular and for square membranes this can be done analytically [2] and for more complicated shapes (and for different boundary conditions at the membrane edges) one can use the 
finite-element methods. However, we can obtain valuable information directly from Eq. (1). Firstly, as the $a$ and $b$ potentials have the same signs we immediately note that the light-hole lines will move faster than the heavy-hole lines. Secondly, the ratio of the light-hole shift to the heavy-hole shift

$$
\alpha=\frac{\Delta E_{\mathrm{lh}}}{\Delta E_{\mathrm{hh}}}
$$

does not depend on pressure, dimensions of the membrane etc. Therefore, if we determine $\alpha$ experimentally, we can obtain the value of the uniaxial deformation potential $b$ from the value of the hydrostatic potential $a$ :

$$
b=a \frac{2(1-2 \nu)}{1+\nu}\left(\frac{\alpha-1}{\alpha+1}\right),
$$

because the latter is well known from hydrostatic measurements $(a=-8.7 \mathrm{eV}$ in GaAs).

\section{Experimental results}

Let us first look at the room-temperature photoluminescence from a $60 \AA$ well with $\mathrm{Al}_{0.3} \mathrm{Ga}_{0.7}$ As barriers in the middle of a circular membrane (Fig. 2). The

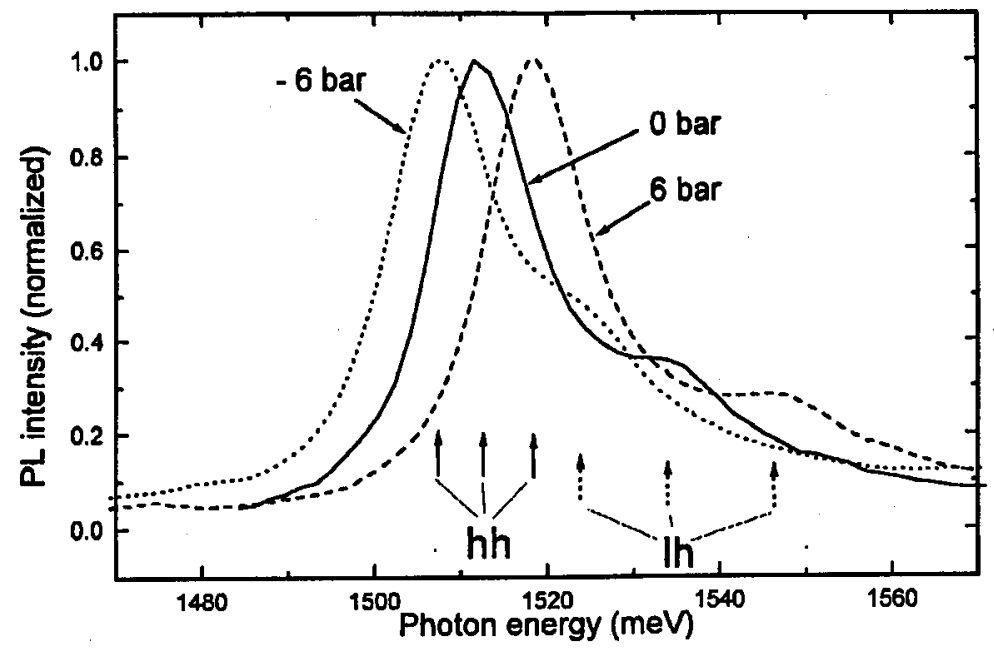

Fig. 2. Shift of the $\mathrm{PL}$ spectra at $300 \mathrm{~K}$ from a $60 \AA$ well with $\mathrm{Al}_{0.3} \mathrm{Ga}_{0.7} \mathrm{As}$ barriers. The laser spot was in the middle of a membrane with $1.26 \mathrm{~mm}$ diameter and $40 \mu \mathrm{m}$ thickness. Arrows show the positions of the heavy and light-hole lines.

light-hole line is rather weak but still we can see that the shift of this line is much stronger than that of the heavy-hole line. For a better resolution we took the sample with wide wells (and, therefore, small heavy-hole light-hole splitting) and we measured photoluminescence (PL) at low temperature $(80 \mathrm{~K})$. In the bottom of Fig. 3 we see the spectrum for zero pressure and then its evolution due 


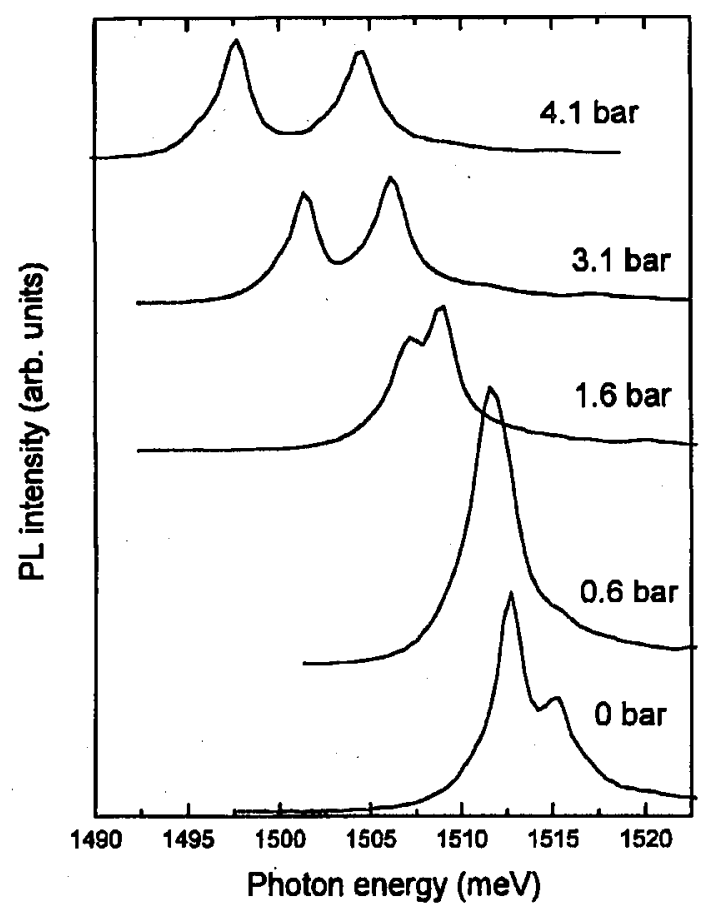

Fig. 3. Shift of the PL spectra at $80 \mathrm{~K}$ from a $250 \AA$ well with $\mathrm{Al}_{0.3} \mathrm{Ga}_{0.7}$ As barriers. The laser spot was in the middle of a membrane with $2 \mathrm{~mm}$ diameter and $30 \mu \mathrm{m}$ thickness. Crossing of the heavy and light-hole lines occurs around 0.6 bar.

to tensile biaxial strain. The two lines clearly cross around $0.6 \mathrm{bar}$ and then they separate, the light-hole line becoming the ground state of the system. The spectra are in scale and one can see that the heavy-hole transitions are stronger than the light-hole ones (the oscillator strength should be 3 times stronger for the heavy holes). The positions of the two peaks are plotted versus pressure in Fig. 4 and the ratio of the pressure coefficients for the light and for the heavy holes obtained from this figure is $\alpha=2.12$. This gives us (through Eq. (3) with $\nu=0.31$ ) the uniaxial deformation potential $b=-1.8 \mathrm{eV}$. The crossover between light-hole line and the excited transitions of the heavy-hole in the photoluminescence excitation (PLE) under compressive uniaxial stress has been reported in [3].

The second example of our method is given in Fig. 5 where we show the absorption from the InGaAs/GaAs quantum well in the middle of a circular membrane. Three lines can be seen in the spectra and the middle one clearly has much stronger pressure shift than the two others. This allows for the unambiguous identification of this line as the light-hole ground state transition lh1. The third line has to be the hh2 transition. We studied this sample under hydrostatic pressure and also as a function of temperature but all the three lines shifted in the same way. Biaxial deformation allows to distinguish between heavy and light-hole lines. This can be helpful in view of some controversies concerning the band offsets in 


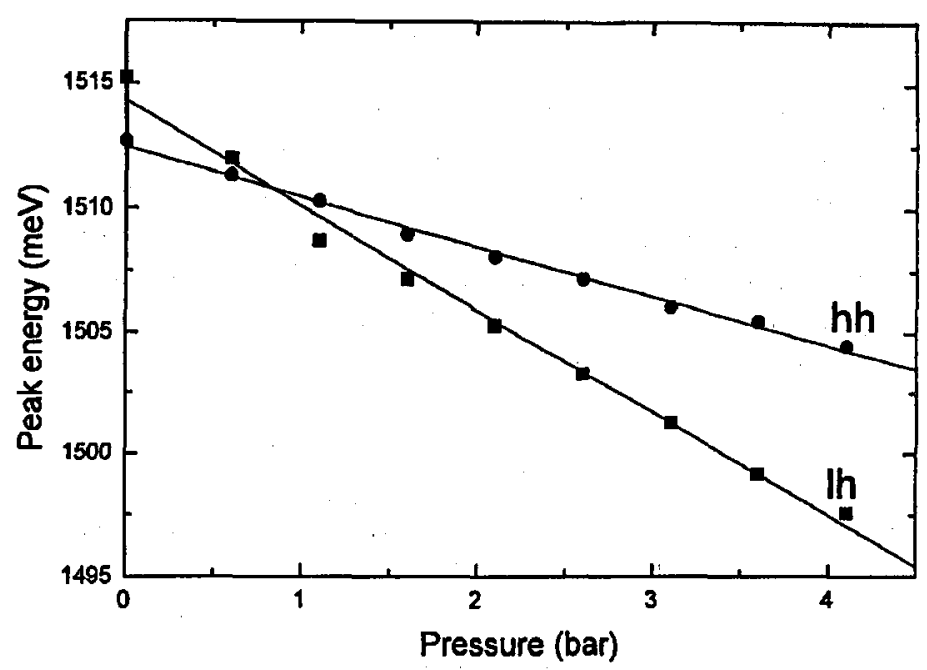

Fig. 4. Position of the peaks from Fig. 3 versus pressure.

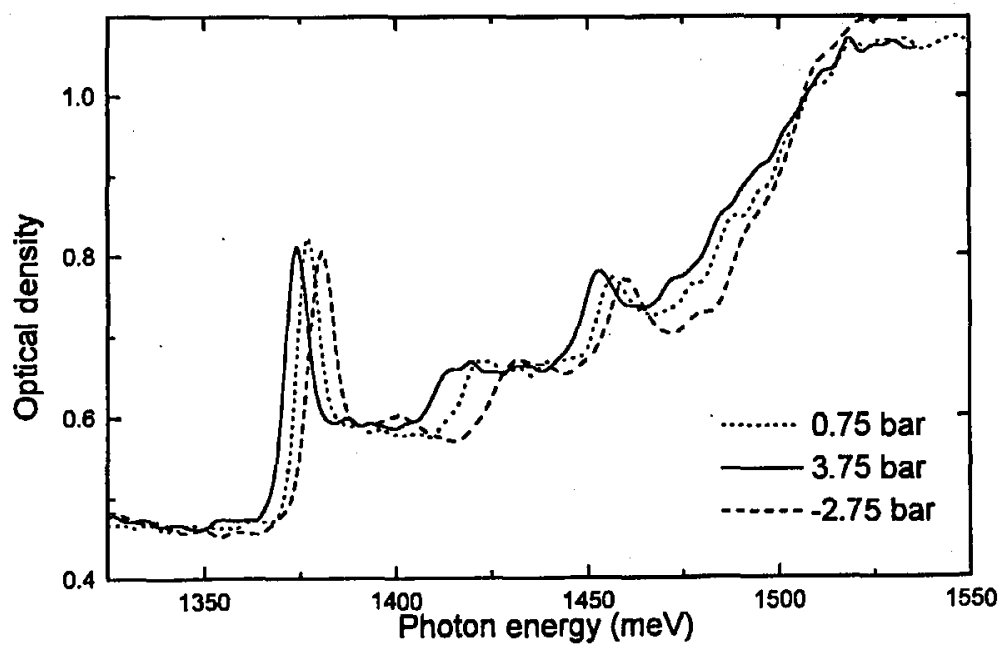

Fig. 5. Absorption spectra at $80 \mathrm{~K}$ from a $100 \AA \operatorname{In}_{0.15} \mathrm{Ga}_{0.85}$ As well with GaAs barriers measured in the middle of a membrane $38 \mu \mathrm{m}$ thick and $3 \mathrm{~mm}$ in diameter.

the InGaAs/GaAs system. The small excitonic effect for the middle line indicates that the light holes are weakly bound. This is consistent with the fact that the valence-band offset is lower than the heavy-light hole splitting induced by the built-in strain. 


\section{Conclusions}

We developed a simple method for obtaining large deformations on the surface of the semiconductor. Contrary to uniaxial and hydrostatic experiments our method does not require sophisticated apparatus. We studied photoluminescence and absorption in deformed quantum wells. Our method can be used for determination of the deformation potentials. It helps to identify heavy-hole and light-hole lines in the spectra. The tunable biaxial deformation can be useful for modeling the effects of built-in strain in lattice mismatched structures.

\section{Acknowledgment}

We acknowledge the financial support of the State Committee for Scientific Research (Republic of Poland), grant number 773369203.

\section{References}

[1] G.L. Bir, G.E. Pikus, Symmetry and Strain-Induced Effects in Semiconductors, Wiley, New York 1974.

[2] S.P. Timoshenko, Theory of Plates and Shells, McGraw-Hill, New York 1959.

[3] Johnson Lee, C. Jagannath, M.O. Vassell, E.S. Koteles, Phys. Rev. B 37, 4164 (1988). 